\title{
Nuevo registro de Oryctanthus grammatus (Loranthaceae), una planta parásita conocida únicamente en su colección tipo
}

\section{New record of Oryctanthus grammatus (Loranthaceae), a parasitic plant known only from the type collection}

\author{
(D) Isabel Carmona-Gallego ${ }^{1, *}$, (D) Jhon Steven Murillo-Serna ${ }^{1}$, \\ Francisco Javier Roldán-Palación ${ }^{2}$, (D) Fernando Alzate-Guarín ${ }^{1}$ \\ ${ }^{1}$ Grupo de Estudios Botánicos, Universidad de Antioquia, Medellín, Colombia \\ ${ }^{2}$ Herbario Universidad de Antioquia, Medellín, Colombia
}

\begin{abstract}
Resumen
Oryctanthus grammatus es una especie ya descrita en el 2011 en el área biogeográfica del río Magdalena, Colombia, de la cual se conoce únicamente su espécimen tipo, recolectado en 1916 en el departamento de Bolívar. En el presente estudio se reporta un espécimen adicional, encontrado en el departamento de Antioquia 71 años después de su primera recolección, y que constituye el segundo registro para la especie. Se discuten brevemente las afinidades taxonómicas de esta especie y se resalta la importancia de las colecciones históricas para el conocimiento de la biodiversidad.
\end{abstract}

Palabras clave: colecciones biológicas; flora de Antioquia; flora de Colombia; Loranthaceae; Santalales.

\begin{abstract}
Oryctanthus grammatus is a species described in 2011 and found in the biogeographic area of the Magdalena River, Colombia, which is known only from the type specimen collected in 1916 in the Department of Bolívar. We report here an additional specimen found 71 years later in the Department of Antioquia, which makes it the second record of this species. We discuss here the taxonomic affinities of the species and we emphasize the value of historical collections for the knowledge of biodiversity.
\end{abstract}

Keywords: Biological collections; flora of Antioquia; flora of Colombia; Loranthaceae; Santalales.

\section{Introducción}

Oryctanthus (Griseb.) Eichler (Loranthaceae) está compuesto por 15 especies que se distribuyen desde el sur de México y Jamaica hasta Bolivia y el norte de Brasil (Kuijt \& Hansen, 2015; Kuijt, 2019). Este género se encuentra representado en Colombia por nueve especies que se distribuyen en todas las regiones biogeográficas del país (Kuijt, 1992; Dueñas, 2016).

El género ha sido estudiado por autores como Eichler (1868), Engler (1897), Kuijt (1976, 1992) y Caires (2012), y en los últimos años se han propuesto cambios de nomenclatura y de circunscripción del género, y se han descrito nuevas especies (Kuijt, 1991, 2009; 2011; Caires, 2012).

Las poblaciones conocidas de algunas especies del género son escasas y muy pocas veces se han recolectado individuos. Entre estas se cuentan Oryctanthus costulatus, O. grandis, O. minor y O. neurophyllus (Caires, 2012), pero de otras especies solo se conoce la del tipo, como es el caso de O. grammatus (Kuijt, 2011), una planta parásita de Colombia que es fácilmente diferenciable por sus características morfológicas (Kuijt, 2011). En este estudio se registra por primera vez en el departamento de Antioquia esta especie, lo que constituye, además, el segundo registro conocido para el taxón. Asimismo, se describe y se ilustra el ejemplar testigo de este nuevo registro y se discute la relevancia de las colecciones antiguas para el estudio de la biodiversidad.

\section{Materiales y métodos}

Durante una revisión del género Oryctanthus (Griseb.) Eichler en Antioquia, se analizaron los protólogos, las revisiones y los especímenes tipo en herbarios virtuales y en la plataforma plants.jstor.org, con el fin de hacer una correcta determinación de los especímenes.

\footnotetext{
*Correspondencia:

Isabel Carmona-Gallego; isabelcg04@gmail.com

Recibido: 21 de junio de 2019

Aceptado: 16 de agosto de 2019

Editor: Elizabeth Castañeda
} 
Las revisiones permitieron encontrar el pliego HUA 53214, determinado como Oryctanthus sp., ejemplar que corresponde a Oryctanthus grammatus Kuijt, y constituye el segundo registro conocido para la especie. El espécimen fue descrito e ilustrado, y se discutieron sus afinidades taxonómicas con especies morfológicamente cercanas y con otras poco conocidas del género Oryctanthus.

Los herbarios custodios de los especímenes tratados en esta nota se referencian según los acrónimos de Thiers (2019).

\section{Resultados}

Oryctanthus grammatus Kuijt. Novon. 21(4): 463. 2011.

TIPO: Colombia. Bolívar: Lands of Loba, San Martín de Loba \& vic., "on mangle” Abril-Mayo 1916, H.M. Curran s.n. (holotipo, US-537561 [imagen digital!]).

Planta hemiparásita, laxamente ramificada. Ramas erectas o péndulas; tallo tri- o tetra-angulado; entrenudos de 5-15 (-22) mm de largo, entrenudo basal normalmente de mayor longitud que los restantes; ejes jóvenes totalmente cubiertos por corcho pustular de color marrón, que luego se limitan a las aristas de los ángulos, tornándose más o menos glabros cuando maduros; haustorio no observado. Hojas opuestas, a veces subopuestas, cartáceas; pecíolo de 2-3 $\mathrm{mm}$ de longitud, claramente diferenciable. Lámina foliar ampliamente ovada a elíptica, simétrica, 20-40 × 5-20 $\mathrm{mm}$, a veces mucho menor, base cuneada u obtusa, ápice redondeado, esclerénquima foliar notoriamente visible. Venación pinnada, 5 venas principales claramente visibles, impresas en el haz y el envés, vena media elevada en el envés, que a veces no alcanza el ápice; arista y márgenes ligeramente ferruginosas. Monoicas. Inflorescencia en espiga indeterminada, axilar, solitaria, raramente 3 , que alcanza hasta $6 \mathrm{~mm}$ de longitud, pedúnculo hasta $1 \mathrm{~mm}$ de longitud. Flores hasta 20 en 4 series, monadas dispuestas en pares dísticos, hermafroditas, sésiles y oblicuas, de cerca de $3 \mathrm{~mm}$ de largo, calículo entero, 6 pétalos, dimórficos, de 1,2-1,5 $\mathrm{mm}$ de largo, rojizos, estambres epipétalos, filamentos inconspicuos, anteras dispuestas en 2 niveles, en o arriba de la mitad de los pétalos, conectivo romo, 4 sacos polínicos, dehiscencia longitudinal, estilo recto, de cerca de $1,4 \mathrm{~mm}$ de longitud. Estigma capitado, ovario cónico, completamente incluido dentro de la cavidad del raquis, $1 \mathrm{~mm}$ de longitud incluido el calículo. Fruto ovoide, liso, de $7 \mathrm{~mm}$ de longitud y $4 \mathrm{~mm}$ de diámetro. Semilla ovoide de $4 \mathrm{~mm}$ de longitud y $2 \mathrm{~mm}$ de diámetro. Embrión dicotiledóneo de $3 \mathrm{~mm}$ de largo.

Nuevo registro: Colombia: Antioquia: Remedios: Carretera Remedios - Zaragoza, cerro Cabezas, km 18, 280 m s.n.m. septiembre, 1987. F.J. Roldán, et al. 812 (HUA, NY). (Figura 1).

\section{Discusión}

El espécimen tipo de Oryctanthus grammatus Kuijt fue recolectado por el botánico estadounidense Hugh McCullum Curran (1875 - 1960) durante los meses de abril y mayo de
1916 cuando realizó su viaje a la localidad de San Martín de Loba, municipio ubicado en el departamento de Bolívar, Colombia (Curran, 1929; Blake \& Atwood, 1942).

Esta especie permaneció desconocida para la ciencia durante casi 100 años, hasta que fue descrita en el 2011 por el botánico canadiense Job Kuijt (1930 - ), quien en la publicación resalta que de la especie se conoce únicamente el espécimen tipo, el cual se encuentra depositado en el herbario US (Kuijt, 2011).

Durante septiembre de 1987, en el marco del proyecto "Flora de Antioquia" (Albert de Escobar, 1985; Callejas, 2013), se recolectó O. grammatus en el municipio de Remedios, ubicado en el nordeste del departamento de Antioquia. El espécimen recolectado se determinó como Oryctanthus sp. hasta el año 2019, en el que se realizó una revisión exhaustiva de los especímenes del género Oryctanthus depositados en el Herbario de la Universidad de Antioquia (HUA). Duplicados de esta colección se encuentran en los herbarios K, MO y NY. Hasta la fecha, el

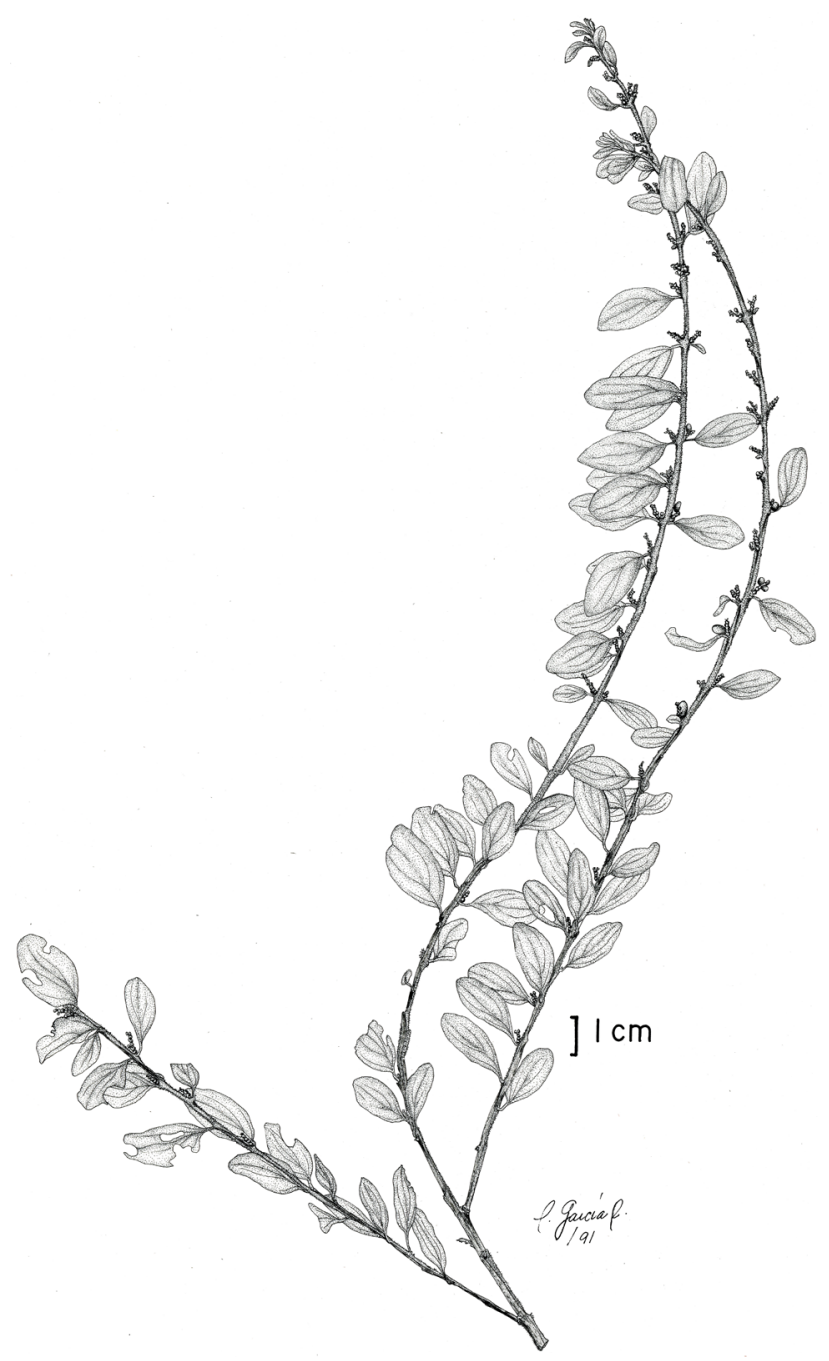

Figura 1. Ilustración de Oryctanthus grammatus Kuijt basada en la colección HUA53214 
pliego de MO se encuentra determinado como Oryctanthus sp. (Missouri Botanical Garden, 2019), en tanto que los duplicados de K y NY son tratados en Caires (2012) como Oryctanthus florulentus (Rich.) Tiegh. Este autor sugiere que $O$. grammatus podría ser un morfotipo de $O$. florulentus. Según él, esta es una especie muy variable en su morfología y ampliamente distribuida en Suramérica, aunque el autor no discute a profundidad la identidad de $O$. grammatus.

En cuanto a las afinidades morfológicas de $O$. grammatus, Kuijt (2011), propone su afinidad con Oryctanthus minor Kuijt, la cual es endémica de la Guyana Francesa. En opinión de Caires (2012), O. minor podría considerarse como un sinónimo de $O$. florulentus con base en la variación exhibida por esta especie.
Teniendo en cuenta las afinidades morfológicas de $O$. grammatus ya expuestas, esta especie puede confundirse con la ampliamente distribuida $O$. florulentus, o con otras especies de inflorescencias cortas como $O$. guianensis Kuijt, $O$. phthirusoides Rizzini y O. minor; y aunque la dificultad para identificar las especies del género Oryctanthus ya ha sido discutida por Caires (2012), O. grammatus claramente se diferencia de estas especies en caracteres morfológicos de fácil observación, además, su distribución geográfica es restringida. La combinación de estos atributos se presenta en la tabla 1. Sin embargo, la discusión queda abierta con respecto al número de especies, el rango de variación y las afinidades taxonómicas en Oryctanthus, por lo que se hace necesario hacer análisis con técnicas moleculares para

Tabla 1. Caracteres morfológicos evaluados en especímenes herborizados que permiten diferenciar O. grammatus de especies similares

\begin{tabular}{|c|c|c|c|c|c|}
\hline Especie & O. grammatus & O. guianensis & O. florulentus & O. phthirusoides & o. minor \\
\hline Longitud del pecíolo & $\simeq 2-3 \mathrm{~mm}$ & $\simeq 5 \mathrm{~mm}$ & $\simeq 3-6 \mathrm{~mm}$ & $\simeq 2-3 \mathrm{~mm}$ & $\simeq 1-2 \mathrm{~mm}$ \\
\hline Textura de la hoja & Cartácea & Coriácea & Coriácea & Coriácea & Crasa \\
\hline $\begin{array}{l}\text { Forma y dimensión de } \\
\text { la hoja }\end{array}$ & $\begin{array}{l}\text { Ampliamente } \\
\text { obovadas a elípticas, } \\
2-4 \times 0,5-2 \mathrm{~cm}\end{array}$ & Elípticas, $5 \times 2,5 \mathrm{~cm}$ & $\begin{array}{l}\text { Lanceoladas, } \\
4-10 \times 1,5-3,5 \mathrm{~cm}\end{array}$ & $\begin{array}{l}\text { Orbiculares y } \\
\text { ligeramente } \\
\text { emarginadas, } \\
1,5-2 \times 1,5-2 \mathrm{~cm}\end{array}$ & $\begin{array}{l}\text { Ovadas-elípticas, } \\
3-4,5 \times 1-2 \mathrm{~cm}\end{array}$ \\
\hline Venación primaria & $\begin{array}{l}\text { Pinnada, a veces la } \\
\text { vena media no alcanza } \\
\text { el ápice }\end{array}$ & $\begin{array}{l}\text { Pinnada, la vena } \\
\text { media siempre } \\
\text { alcanza el ápice }\end{array}$ & $\begin{array}{l}\text { Pinnada, la vena } \\
\text { media siempre alcanza } \\
\text { el ápice }\end{array}$ & Palmeadas & $\begin{array}{l}\text { Pinnada, la vena } \\
\text { media siempre } \\
\text { alcanza el ápice }\end{array}$ \\
\hline Venación secundaria & Claramente visible & Poco visible & Claramente visible & $\begin{array}{l}\text { Poco visible, se } \\
\text { confunde con el } \\
\text { esclerénquima foliar }\end{array}$ & Poco visible \\
\hline $\begin{array}{l}\text { Indumento corchoso } \\
\text { furfuráceo en las hojas }\end{array}$ & $\begin{array}{l}\text { Muy tenue, en la vena } \\
\text { media }\end{array}$ & $\begin{array}{l}\text { En la vena media } \\
\text { muy conspicuo, en } \\
\text { pecíolo y margen de } \\
\text { la hoja, presente }\end{array}$ & $\begin{array}{l}\text { Tenue en la vena } \\
\text { media y en el margen } \\
\text { de la hoja }\end{array}$ & Casi ausente & $\begin{array}{l}\text { Muy conspicuo } \\
\text { en la vena } \\
\text { media, pecíolo } \\
\text { y margen foliar }\end{array}$ \\
\hline Esclerénquima foliar & Muy evidente & No visible & Usualmente visible & Muy evidente & No visible \\
\hline Forma de los tallos & $\begin{array}{l}\text { Angulosos, delgados y } \\
\text { estilizados }\end{array}$ & $\begin{array}{l}\text { Angulosos, con } \\
\text { grosor variable }\end{array}$ & $\begin{array}{l}\text { Angulosos, con grosor } \\
\text { variable }\end{array}$ & Teretes & Teretes \\
\hline $\begin{array}{l}\text { Indumento corchoso } \\
\text { furfuráceo en los tallos } \\
\text { jóvenes }\end{array}$ & $\begin{array}{l}\text { Denso en los primeros } \\
\text { entrenudos, luego solo } \\
\text { en los ángulos }\end{array}$ & En los ángulos & En los ángulos & Muy tenue & $\begin{array}{l}\text { Cubriendo la } \\
\text { totalidad del eje }\end{array}$ \\
\hline $\begin{array}{l}\text { Longitud de los } \\
\text { entrenudos }\end{array}$ & $\begin{array}{l}5-15(-22) \mathrm{mm}, \\
\text { usualmente }<15 \mathrm{~mm}\end{array}$ & $15-40 \mathrm{~mm}$ & $10-45 \mathrm{~mm}$ & $5-15 \mathrm{~mm}$ & $5-20 \mathrm{~mm}$ \\
\hline Longitud del pedúnculo & $<1 \mathrm{~mm}$ & $<3 \mathrm{~mm}$ & $<2 \mathrm{~mm}$ & $<2 \mathrm{~mm}$ & $<2 \mathrm{~mm}$ \\
\hline $\begin{array}{l}\text { Numero de ejes florales } \\
\text { por axila foliar y } \\
\text { longitud }\end{array}$ & $\begin{array}{l}1(-3) \text { ejes, hasta } 6 \\
\mathrm{~mm}\end{array}$ & $1 \mathrm{eje}$, hasta $20 \mathrm{~mm}$ & $1-4$ ejes, hasta $50 \mathrm{~mm}$ & 1 eje, hasta $10 \mathrm{~mm}$ & $\begin{array}{l}1-3 \text { ejes, hasta } \\
10 \mathrm{~mm}\end{array}$ \\
\hline $\begin{array}{l}\text { Número de flores por } \\
\text { eje floral y orientación } \\
\text { con respecto al eje }\end{array}$ & $\begin{array}{l}\text { Hasta } 20 \text { flores, } \\
\text { oblicuas }\end{array}$ & $\begin{array}{l}\text { 12-24 flores, } \\
\text { perpendiculares }\end{array}$ & $\begin{array}{l}\text { Más de } 20 \text { flores, } \\
\text { oblicuas }\end{array}$ & 6-28 flores, oblicuas & $\begin{array}{l}12-40 \text { flores, } \\
\text { oblicuas }\end{array}$ \\
\hline $\begin{array}{l}\text { Distribucion geográfica } \\
\text { conocida }\end{array}$ & $\begin{array}{l}\text { Colombia, Valle del } \\
\text { Magdalena Medio, } \\
\text { departamentos de } \\
\text { Antioquia y Bolívar } \\
\text { (localidad tipo) }\end{array}$ & Guyana Francesa. & $\begin{array}{l}\text { Suramérica: Surinam, } \\
\text { Guyana, Guyana } \\
\text { Francesa, Brasil, } \\
\text { Venezuela, Colombia, } \\
\text { Ecuador, Perú, Bolivia }\end{array}$ & $\begin{array}{l}\text { Amazonia: Brasil, } \\
\text { Venezuela y } \\
\text { Colombia }\end{array}$ & Guyana Francesa \\
\hline
\end{tabular}


recabar evidencia adicional que soporte las hipótesis propuestas con base en los caracteres morfológicos, o que, por el contrario, suministre una nueva visión con respecto a los límites específicos del género y su riqueza.

La especie Oryctanthus grammatus Kuijt es endémica de Colombia y hasta la fecha solo se conocen especímenes provenientes de dos localidades en los departamentos de Bolívar y Antioquia (Figura 2). Ambas localidades se encuentran en el área biogeográfica del Valle del Magdalena, el cual corresponde al ecosistema potencial de bosque húmedo tropical (Etter, et al., 2006). Infortunadamente, este territorio ha sido uno de los más explotados comercialmente en Colombia, lo que ha provocado un deterioro notable de sus áreas naturales (Etter, et al., 2006, 2008). Por estas razones, cuando Kuijt (2011) describió O. grammatus sugirió que la especie podría estar extinta. Aun así, debido a la falta de registros para asignarle un área, la especie es asignada a la categoría DD (datos insuficientes), según los criterios de la International Union for Conservation of Nature (International Union for Conservation of Nature

- IUCN, 2012, 2014).

El descubrimiento de una nueva aparición en los años 80 sugeriría que $O$. grammatus no es un taxón extinto y que constituye una especie rara o poco recolectada, tal vez debido a su hábito poco llamativo y flores diminutas (Kuijt, 2011). Sin embargo, es importante resaltar que estas recolecciones se realizaron hace más de 30 años y que en el departamento de Antioquia se ha registrado una pérdida acelerada de áreas naturales (González-Caro \& Vásquez, 2017). Por todo ello, deben explorarse exhaustivamente estas zonas para obtener información básica que permita conocer el estado de conservación de esta especie.

El redescubrimiento de esta especie rara o poco recolectada es un claro ejemplo del valor que tienen las colecciones biológicas para el conocimiento de la biodiversidad y su contribución potencial para establecer prioridades de

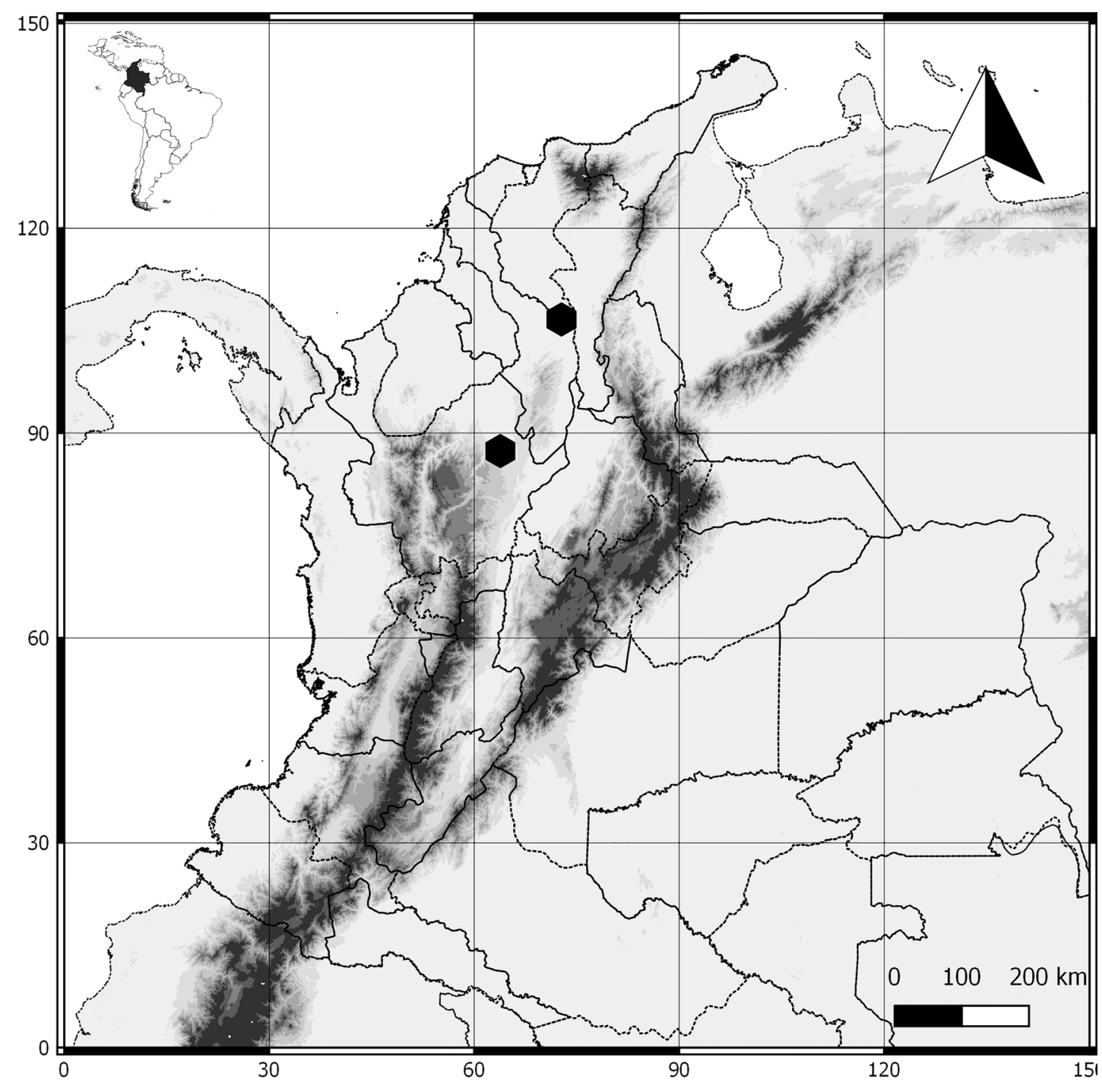

Figura 2. Distribución conocida de Oryctanthus grammatus Kuijt 
conservación en regiones biodiversas, un tema ampliamente discutido y que cada vez adquiere una mayor rele-vancia (Granados-Tochoy, et al., 2007; Loiselle, et al., 2008; Nualart, et al., 2017).

La digitalización de las recolecciones antiguas o históricas permite tener acceso masivo y ágil a estas para conocer la biodiversidad existente en diferentes áreas del planeta (Meineke, et al., 2018). Los estudios basados en colecciones biológicas hoy pueden tardar menos gracias a las colecciones digitales, y aunque estas no son comparables con los especímenes en físico, las bases de datos en línea suministran la información para abordar muchas preguntas en diferentes áreas del conocimiento (Nelson \& Ellis, 2018).

\section{Conclusiones}

Oryctanthus grammatus se puede diferenciar por sus características morfológicas, sin embargo, queda abierta la discusión sobre su estatus taxonómico en el género. Por esto, se requieren análisis adicionales que aporten información útil para esclarecer el número de especies del género, así como sus límites y afinidades morfológicas.

El estudio detallado de las colecciones de herbario constituye un importante insumo para conocer la biodiversidad regional y en los ecosistemas. Además, las colecciones históricas representan fuentes de información que aportan datos sobre la extinción y el estado de conservación de las especies. Para conocer estos aspectos de la biología de las especies y la dinámica de los ecosistemas, la digitalización de las colecciones biológicas con acceso al público representa una muy importante herramienta para el estudio de la diversidad, ya que facilita su divulgación y la consulta rápida y amplia.

\section{Contribuciones de los autores}

FJRP, ICG, JSMS confirmaron la identidad taxonómica del nuevo registro. ICG, JSMS, FJRP y FAG consultaron el material de herbario y elaboraron el manuscrito.

\section{Conflicto de intereses}

Los autores declaran no tener conflicto de intereses.

\section{Agradecimientos}

Los autores expresan sus agradecimientos al personal del herbario HUA por facilitar el acceso a sus equipos e instalaciones, a Consuelo García por la ilustración del espécimen y a Mauricio Posada por suministrar bibliografía relevante y fotografías del espécimen depositado en el herbario NY.

\section{Material suplementario}

Especímenes examinados. Oryctanthus florulentus (Rich.) Tiegh. Colombia. Antioquia: Mpio. Puerto Triunfo, "Corregimiento Doradal, cerca de la Cueva del Cóndor" Roldán F. \& Betancur J. 719 (HUA); Mpio. San luis, "Río Samaná norte, margen izquierda del río, sobre la vía Medellín-Bogotá" Callejas R., et al. 4092 (HUA); Mpio. Sonsón, "Jurisdicción de Cementos ARGOS, sector El Billar, subiendo por los potreros de Luis Cardona" David H. et al. 4078 (HUA). Choco: "Hoya del río San Juan, alrededores de Palestina" Forero E., et al. 4018 (HUA); "Hoya del río San Juan, Andagoya, campamento de la Cia. Mineros del Chocó" Forero E., et al. 5114 (HUA); "Hoya del río San Juan, quebrada Cunperro, margen izquierda del río San Juan, debajo de Noanamá" Forero E., et al. 4864 (HUA). Valle Del Cauca: Mpio. Buenaventura, "Trayecto Córdoba-San Cipriano" Ramírez B., et al. 12468 (HUA); Mpio. Buenaventura, "Vereda Bendiciones, parador Los Chorrillos, al pie de la quebrada Tomines, km 37 Cali-Buenaventura" Ramírez B., et al. 12434 (HUA). Vaupés: Mpio. Mitú, "Along bank of río Vaupés” Zarucchi J., et al. 1644 (HUA). Ecuador. Pastaza: Mpio. Pastaza, "Población de Moretococha" Gudiño E., et al. 1381 (HUA). Guyana. Islas Del Esequibo-Demerara Occidental: "Naamryck Canal, +/- $8 \mathrm{~km}$ SE of Naamryck Public Rd, just W of Lookout village" Pipoly J., et al. 11277 (HUA); — sin localidad precisa, Leblond J.B. s.n. [imagen digital!] (G). Oryctanthus guianensis Kuijt. Guyana. Cayena: Kourou, "Garden" Billiet F. \& Jadin B. 5923 [imagen digital!] (BR, MO). Oryctanthus minor Kuijt. Guyana. "Monts Bakra, à 1,5 km à l'ouest Pic Coudreau" Granville J., et al. 14899 [imagen digital!] (UC). Oryctanthus phthirusoides Rizzini. Brasil. Amazonas: "Lauareté, Margem do Rio Uaupés." Ribeiro B.G.S. 998 [imágenes digitales!] (IAN, RB). Colombia. Caquetá: Mpio. Solano, "Margen izquierda del río Caquetá, área del Caño Paujil, $10 \mathrm{Km}$ al NO de la Araracuara" Arbeláez M. \& Sueroque F. 525 (HUA). Vea el material suplementario en doi: http:// dx.doi.org/10.18257/raccefyn.921

\section{Referencias}

Albert de Escobar, L. (1985). Notas del Herbario: Programa "Flora de Antioquia". Act Biol. 14: 105-106.

Blake, S.F. \& Atwood, A.C. (1942). Geographical guide to floras of the world: An annotated list with special reference to useful plants and common plant names, Part I. Washington, United States: United States Government Printing Office. p. 336.

Caires, C.S. (2012). Estudos taxonômicos aprofundados de Oryctanthus (Griseb.) Eichler, Oryctina Tiegh. e Pusillanthus Kuijt (Loranthaceae). (Tesis de doctorado). Brasilia: Brasil. Universidade de Brasília, Instituto de Ciências Biológicas, Departamento de Botânica.

Callejas, R. (2013). La Exploración Botánica en el Departamento de Antioquia (1808-2000). En: R. Callejas, A. Idárraga. Flora de Antioquia: Catálogo de las Plantas Vasculares. Vol. I. p. 293-326. Bogotá, Colombia: Editorial D’Vinni.

Curran, H.M. (1929). The Lands of Loba. Trop Woods, 19: 11-38.

Dueñas, H. del C. (2016). Loranthaceae. En: R. Bernal, S.R. Gradstein, M. Celis. Catálogo de plantas y líquenes de Colombia, p. 1487-1493. Bogotá, Colombia: Universidad Nacional de Colombia (Sede Bogotá). Facultad de Ciencias. Instituto de Ciencias Naturales.

Eichler, A.W. (1868). Loranthaceae. En: K. von Martius. Flora Brasiliensis, p. 87-92. Leipzig, Alemania.

Engler, A. (1897). Loranthaceae. En: A.W. Engler, K. Prantl. Die natürlichen Pflanzenfamilien, Nachträge III, p. 124-140. Leipzig, Alemania: Verlag von Wilhelm Engelmann. 
Etter, A., McAlpine, C., Wilson, K., Phinn S., Possingham, H. (2006). Regional patterns of agricultural land use and deforestation in Colombia. Agric Ecosyst Environ. 114 (24): 369-386. Doi: 10.1016/j.agee.2005.11.013

Etter A., McAlpine C., Possingham, H. (2008). Historical patterns and drivers of landscape change in Colombia since 1500: A regionalized spatial approach. Ann Assoc Am Geogr. 98 (1): 2-23. Doi: 10.1080/00045600701733911

González-Caro, S. \& Vásquez, A. (2017). Estado de los bosques de Antioquia entre 1990-2015. En: E. Quintero-Vallejo, A.M. Benavides, N. Moreno, S. Gonzalez-Caro. Bosques Andinos, estado actual y retos para su conservación en Antioquia, p. 64-80. Medellín, Colombia: Fundación Jardín Botánico de Medellín Joaquín Antonio Uribe-Programa Bosques Andinos (COSUDE).

Granados-Tochoy, J.C., Knapp, S., Orozco, C.I. (2007). Solanum humboldtianum (Solanaceae): An endangered new species from Colombia rediscovered 200 years after its first collection. Syst Bot. 32 (1): 200-207. Doi: 10.1600/036364407780360085

International Union for the Conservation of Nature - IUCN. (2012). IUCN red list categories and criteria: Version 3.1. Second edition. IUCN, Gland, Switzerland and Cambridge, UK.

IUCN Standards and Petitions Subcommittee. (2014). Guidelines for using the IUCN red list categories and criteria. Version 11. Prepared by the standards and petitions subcommittee. Fecha de consulta: 15 de junio de 2019. Disponible en: http://www.Iucnredlist.Org/documents/

Kuijt, J. (1976). Revision of the genus Oryctanthus (Loranthaceae). Bot Jahrb. 95: 478-534.

Kuijt, J. (1991). Inflorescence structure and generic placement of some small-flowered species of Phthirusa (Loranthaceae). Syst. Bot. 16 (2): 283-291. Doi: 10.2307/2419280

Kuijt, J. (1992). Nomenclatural changes, new species, and a revised key for the genus Oryctanthus (Loranthaceae). Bot Jahrb. 114: 173-183.
Kuijt, J. (2009). Miscellaneous mistletoe notes, 48-60: Descriptions of twelve new species of Loranthaceae and Viscaceae. Brittonia. 61 (2): 144-162. Doi: 10.1007/s12228-008-9068-2

Kuijt, J. (2011). Two new species of Oryctanthus (Loranthaceae) from Colombia and French Guiana. Novon. 21 (4): 463467. Doi: $10.3417 / 2010106$

Kuijt, J. \& Hansen, B. (2015). Loranthaceae. En: K. Kubitzki. Flowering Plants Eudicots: Santalales, Balanophorales, p. 73-119. Springer International Publishing.

Kuijt, J. (2019). New World Mistletoes - Oryctanthus (Griseb.) Eichler. Fecha de consulta: junio de 2019. Disponible en: http://www.tropicos.org/Name/40034298?projectid=79

Loiselle, B. A., Jørgensen, P.M., Consiglio, T., Jiménez, I., Blake, J.G., Lohmann, L.G., Montiel, O.M. (2008). Predicting species distributions from herbarium collections: Does climate bias in collection sampling influence model outcomes?. J Biogeogr. 35 (1): 105-116. Doi: 10.1111/j.1365-2699.2007.01779.x

Meineke, E.K., Davies, T.J., Daru, B.H., Davis, C.C. (2018). Biological collections for understanding biodiversity in the Anthropocene. Phil Trans R Soc B. 374 (1763): 2-9. Doi: 10.1098/rstb.2017.0386

Missouri Botanical Garden. (2019). Tropicos. Fecha de consulta: junio de 2019. Disponible en: http://www.tropicos.org/ Specimen $/ 721849$

Nelson, G. \& Ellis, S. (2018). The Impact of Digitization and Digital Data Mobilization on Biodiversity Research and Outreach. BISS. 2: e28470. Doi: 10.3897/biss.2.28470

Nualart, N., Ibáñez, N., Soriano, I., López-Pujol, J. (2017). Assessing the relevance of herbarium collections as tools for conservation biology. Bot Rev. 83 (3): 303-325. Doi: 10.1007/s12229-017-9188-Z

Thiers, B. (2019). Index Herbariorum: A global directory of public herbaria and associated staff. Fecha de consulta: junio de 2019. Disponible en: http://sweetgum.nybg.org/ih/ 